\title{
Analytical-experimental analysis of last generation medium-size motorcycles emission behaviour under real urban conditions
}

\author{
Paolo Iodice* and Adolfo Senatore \\ University of Naples Federico II, Department of Industrial Engineering, \\ Via Claudio 21 - 80125 Napoli, Italy \\ *Email: paolo.iodice@unina.it, \\ Phone: (+39) 081 7683277, Fax: (+39) 0812396097
}

\begin{abstract}
Mopeds and motorcycles are broadly used and represent a large share of motorized vehicles, above all in the urban environment of Asia and Europe; as a consequence, the relative contribution of the emissions of powered two-wheelers to the total air pollution has increased considerably in the last years. For this reason, an experimental investigation was performed on the regulated pollutants: $\mathrm{CO}$ (carbon monoxide), $\mathrm{HC}$ (unburned hydrocarbons), and $\mathrm{NO}_{\mathrm{x}}$ (oxides of nitrogen) emissions were evaluated in the exhaust of a motorcycle with a displacement of $250 \mathrm{~cm}^{3}$, equipped with catalytic converter and electronic mixture control. This vehicle was tested on a dynamometer bench both on a Type-Approval test cycle and on real-world test cycles. The study also presents a method to analyze, during different driving cycles, the influence of the speed profile on the emission factors of the motorcycle, through the elementary kinematic parameters of basic sequences, which were achieved through appropriate fragmentation of composite urban driving cycles. Examination of the results obtained in this study has recognized the kinematic parameters that cause higher $\mathrm{CO}$ and $\mathrm{HC}$ emissions. By using this statistical method, the vehicle emission assessment is meaningfully improved if compared to the models that are mainly centred on the average speed of the test cycles. The statistical processing results of the experimental data were also compared to current emission models for calculating emissions from road traffic in Europe.
\end{abstract}

Keywords: Cold start transient; exhaust emission; motorcycle emission factors and emissive behaviour; real-world test cycles.

\section{INTRODUCTION}

This paper aims to analyze the emissions of the last generation medium-size motorcycles (so-called scooter) under real conditions, contributing to extended knowledge of new sold motorcycles emission behaviour, with reference to cold and hot conditions. Two-wheelers are popular means of transportation, particularly in eastern and southern Asia and in southern Europe, above all in Italy. The great cities of these countries, in fact, host large fleets of these vehicles, helping to meet quotidian urban transport requests [1]; in these urban contexts, the use of these vehicles is linked to several aspects, for example parking simplicity, rapidity in traffic jams, low-cost maintenance. In Italy, powered two-wheelers (including mopeds and motorcycles) cover almost $20 \%$ of all on-road circulating vehicles $[2,3]$. Comparison and appraisal of emissions produced by two-wheelers and passenger cars reveal that the absolute emission level of passenger cars have been reduced significantly in the last two decades, due to the introduction of legislation together with 
tightening the applicable limits on regulated emissions for these vehicles, while on the other hand, the after-treatment technologies used for motorcycles have not been as efficient as those for cars $[4,5]$. Available strategies to control emissions already in use on passenger cars, in fact, are often not used on motorcycles because they prove to be too expensive in relation to vehicle cost and their real effectiveness [6, 7]. As a result, motorcycles and mopeds make a considerable contribution to $\mathrm{CO}$ and $\mathrm{HC}$ although the percentage of two-wheelers in the total circulating fleet is lower than that of other vehicle categories [8]. Determination of emissions from two-wheelers is then very important for estimating the relevant contribution to total emissions attributable to road transport, also taking into account that their impact to air pollution is generally growing, especially in urban environments. For all these concerns, in the last years, experimental investigations on the emissive behaviour of two-wheel vehicles were performed by the Department of Industrial Engineering of the University of Naples Federico II in the Istituto Motori emission laboratory (National Research Council). In this particular study, the experimental activity was performed in order to characterize the emissive behaviour during different driving cycles of a scooter on the basis of roller test bench measurements. $\mathrm{CO}, \mathrm{HC}$ and $\mathrm{NO}_{\mathrm{x}}$ emissions were evaluated in the exhaust of a motorcycle of $250 \mathrm{~cm}^{3}$ swept volume, which belongs to the Euro-3 category (European legislative category for motorcycles, as described in the Directive 97/24/CE). The emission performance of this motorcycle was determined on the statutory driving cycles for Europe ("UDC+EUDC" and "ECE-47") and on different real-world driving cycles: the Worldwide Motorcycle Test Cycle ("WMTC") and the "Artemis Urban Cold" driving cycle.

The current emission models available in Europe for calculating emissions from road traffic are mainly based (especially for the two-wheelers) on fixed legislative driving standards, not on the local driving conditions underestimating cycle dynamics, and do not take into consideration in detail the warm-up behaviour of motorcycles [9]. The emission factors measured in such conditions might not be adequately descriptive of real-world motorcycle riding. For this reason, this study has also developed a method to analyze, during different driving cycles, the influence of speed profile on the $\mathrm{CO}$ and $\mathrm{HC}$ emission factors of this motorcycle through two kinematic factors: the average speed and the product of average speed and average acceleration of basic kinematic sequences. These basic sequences were achieved through appropriate fragmentation of composite urban driving cycles. This study then analyzes the influence of different driving cycles (characterized by different kinematic parameters) on motorcycle emission behaviour during the cold and the hot phases.

The experimental data of this research and the relevant statistical processing results allowed to develop some interesting comparison with current emission models for calculating emissions from road traffic, namely COPERT (Computer Programme to calculate Emissions from Road Transport) and ARTEMIS (Assessment and Reliability of Transport Emission Models and Inventory Systems). The COPERT methodology is the most commonly used model in Europe for official national inventories of emissions from road traffic. The current version is a collaborative effort and draws its main elements from several large-scale European activities: the MEET project, the COST 319 action on the Estimation of Emissions from Transport, and the ARTEMIS project [10]. In the COPERT methodology, emissions of exhaust gases can be estimated for 36 pollutants, and also for many secondary pollutants, the total emissions are calculated by summing emissions from three different sources, namely the thermally stabilized engine operation (hot), the warming-up phase (cold start) and due to evaporation. Since vehicle emissions depend on the engine operation (i.e. driving situation), exhaust emissions are calculated as a function 
of average speed and for three driving conditions: urban, rural and highway. The methodology allows the estimation of the emissions for 230 vehicle categories belonging to the following five main classes: passenger cars, light duty vehicles, heavy duty vehicles, urban buses and coaches, and two wheelers. Vehicles belonging to such main classes are then distinguished according to the fuel type, the EU Directives to which they conform in terms of emissions, the cylinder capacity and other variables. However, regarding the estimation of the emissions of two wheelers vehicle category, the COPERT model reports mainly the values calculated and reported in the ARTEMIS project WP500.

The ARTEMIS project generated an emission model for all transport means and its objective is to offer reliable emission estimates at the sundry levels: regional, national and international. This project contains of 13 work packages; and between these, the WP 500 [11] focuses on emissions of powered two-wheelers. The ARTEMIS WP500 project is centred on a great number of bag and online emission results both from numerous experimental studies and international measurement programme developed during the last years. Almost 2600 emission results were obtained and are accessible to assist as a base for emission modelling. Consequently, the focal target of ARTEMIS WP500 project was to improve a set of typical emission results for carbon monoxide, unburned hydrocarbon and oxides of nitrogen, available for both old and last generation motorized two-wheelers, and that will be the source for the emission models of this vehicle category. The method to define emission levels for motorcycles used in this project was derived from the methodology employed to update the two-wheelers section of the Handbuch emission model (HBEFA) studied by Steven [12].

Table 1. Vehicle categorisation for powered two-wheelers in ARTEMIS WP500.

\begin{tabular}{llll}
\hline Category & Vehicle category & Swept volume $\left[\mathrm{cm}^{3}\right]$ & Engine \\
\hline 1 & moped & vol $<50$ & 2-stroke \\
2 & & & 4 -stroke \\
3 & motorcycle & vol $\leq 150$ & -stroke \\
4 & & & -stroke \\
5 & motorcycle & $150<\mathrm{vol} \leq 250$ & 4-stroke \\
6 & & $250<\mathrm{vol} \leq 750$ & 4 -stroke \\
7 & motorcycle & vol $>750$ & \\
8 & & & \\
\hline
\end{tabular}

Table 1 provides the vehicle categories that were defined for the powered twowheelers. These eight initial categories again need another distinction with regards to legislative category: Euro-0, Euro-1, Euro-2 or Euro-3. Hence, all 32 categories are identified for which emission factors were determined. The measurement data (both online and bags) were employed to derive by regression analysis emission functions in terms of mass per kilometre in relation to vehicle speed, for each powered two-wheeler vehicle category, obtaining fifth order polynomial functions (1) (with $v$ as vehicle speed in $[\mathrm{km} / \mathrm{h}]$ and $\alpha_{0}, \alpha_{1}, \alpha_{2} \alpha_{3} \alpha_{4}$ and $\alpha_{5}$ depending on the specific category). By using these emission functions, emission factors can be predicted for driving patterns or test cycles for which no measurement results are available. The results of ARTEMIS project WP500 for the specific vehicular class examined in this study (Euro-3 legislative category and $250 \mathrm{~cm}^{3}$ swept volume) are presented in the following paragraphs and compared to the experimental emission factors obtained during the experimental campaign. 


$$
E[\mathrm{~g} / \mathrm{km}]=\alpha_{5} \cdot v^{5}+\alpha_{4} \cdot v^{4}+\alpha_{3} \cdot v^{3}+\alpha_{2} \cdot v^{2}+\alpha_{1} \cdot v+\alpha_{0}
$$

\section{MATERIALS AND METHODS}

\section{The Vehicle}

The main characteristics of the motorcycle employed in the test series are summarized in Table 2. This motorcycle is equipped with four-stroke engine and the technology used for pollutants abatement to meet the latest emissive standards is the use of a three-way catalytic converter, which features lambda sensors. A precise tuning of air/fuel ratio is reached throughout an electronic fuel injection and a closed-loop exhaust after-treatment control system is thus assumed to be implemented.

Table 2. Technical specifications of the tested motorcycle.

\begin{tabular}{ll}
\hline Category & Motorcycle \\
Engine principle & 4 -stroke \\
Cubic capacity $\left[\mathrm{cm}^{3}\right]$ & 244 \\
Compression ratio & $11.0: 1$ \\
Fuel system & electronic injection \\
Cooling system & liquid \\
Max power $[\mathrm{kw}]$ & $16.2 @ 8250 \mathrm{rpm}$ \\
Maximum speed $[\mathrm{km} / \mathrm{h}]$ & 125 \\
After-treatment system & catalytic converter \\
Legislative category & Euro 3 \\
\hline
\end{tabular}

\section{The Emission Laboratory}

The motorcycle was tested on a chassis dynamometer (AVL Zollner 20" - single roller) in the Istituto Motori emission laboratory (National Research Council), which allows the reproduction of vehicle masses from small mopeds up to heavy two-wheel vehicles (range around $80-450 \mathrm{~kg}$ ). This bench is planned to simulate the road load (with the vehicle inertia) and to measure the exhaust emissions during speed cycles. By using this chassis dynamometer, it is also possible to perform experimental tests in constant speed mode, constant acceleration mode, and constant tractive force mode. This chassis dynamometer was set by employing the running resistance table agreeing to the techniques adopted in Directive 97/24/EC. A variable speed refrigeration blower is located in front of the vehicle so as to direct the cooling air in a way that reproduces real operating situations. The blower speed is such that the linear velocity of the air at the blower outlet is inside $\pm 5 \mathrm{~km} / \mathrm{h}$ of the equivalent roller speed, and for speeds lower than $10 \mathrm{~km} / \mathrm{h}$, air velocity is set to zero. Before each test in cold start conditions, the scooter is kept at constant temperature between $20^{\circ} \mathrm{C}$ and $25^{\circ} \mathrm{C}$ for at least 8 hours. During the experimental tests, the exhaust gases are diluted with purified air by a Mixing Unit attached to a Constant Volume Sampling with Critical Flow Venturi unit (AVL CFV-CVS). During these tests, a constant sample flow of the mixture fills the bags; in these conditions, the concentrations of carbon monoxide, unburned hydrocarbons, oxides of nitrogen and carbon dioxide are determined as average test values. These average test values and continuous emissions are measured with an exhaust gas analysis system (AVL AMA 4000), agreeing to the procedures adopted and suggested in the Directive 97/24/EC. The exhaust emissions are collected in the dilution tunnel and examined at $1 \mathrm{~Hz}$; besides, the values of continuous emissions are adjusted for the time delay compared to the speed. No other compensation 
(i.e. mixing dynamics) or signal treatment is enforced [13]. Figure 1 shows a scheme of this experimental apparatus.

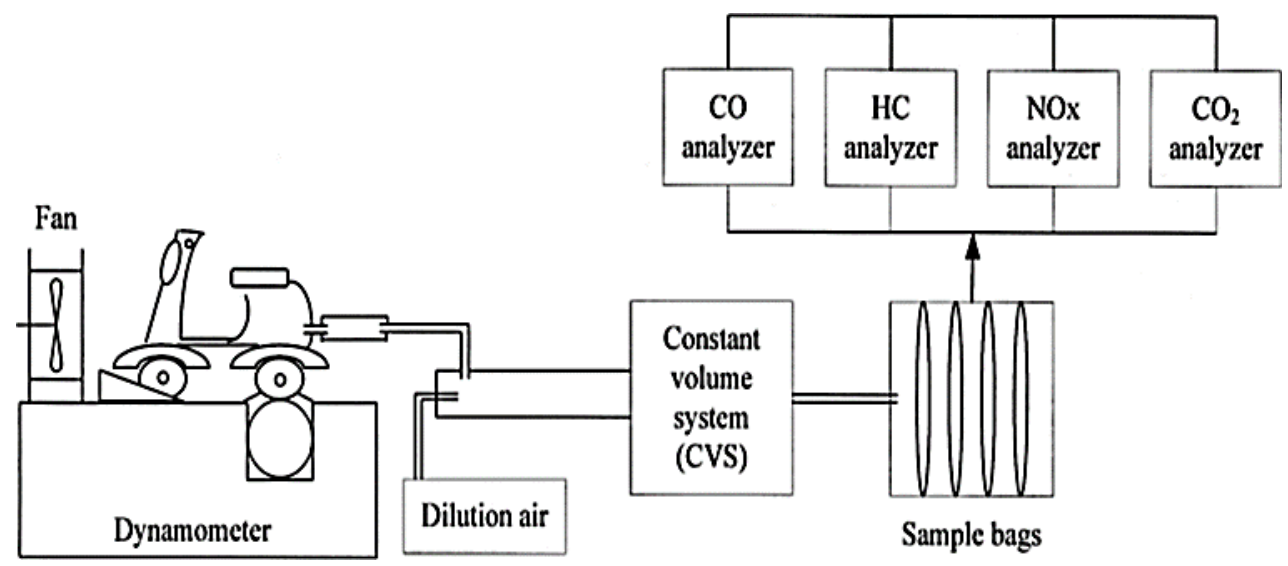

Figure 1. The experimental apparatus of emission laboratory.

\section{Methodologies and Driving Cycles Employed for the Experimental Activities}

Recent emission models available in Europe (and relevant literature) to forecast emission levels are based mainly on the average speed of the driving cycles, and they are not so sensitive to differences of instantaneous speed and acceleration of the vehicles, which definitely affect fuel consumptions and emissions. For this reason, therefore, it is necessary for a complete assessment of the motorcycle emissive behaviour by evaluating the emissions on numerous driving cycles, including several acceleration phases, often requiring rapid changes in vehicle speed; in these phases, an enrichment of the air-fuel mixture is needed, which could affect the catalyst conversion efficiency.

The presented work therefore aims to determine the influence of several driving cycles on emissions of one Euro 3 medium-size motorcycle (so-called scooter). For this purpose, the motorcycle was tested over the following driving cycles with cold start (engine off for at least 6 hours before starting):

i) European Type-Approval driving cycle for motorcycles (ECE+EUDC)

ii) European type-Approval driving cycle for mopeds (ECE-47)

iii) World-wide Motorcycle Emissions Test Cycle (WMTC)

iv) Artemis Urban Cold

"ECE+EUDC" driving cycle is composed of an urban (ECE) and an extra urban part (EUDC); urban part is divided into two phases: cold (UDC_cold including 2 base modules) and hot (UDC_hot including 4 base modules). The "ECE-47" driving cycle comprises of eight elementary cycles; each elementary cycle lasts 112 seconds and includes an acceleration phase and a full speed phase. "WMTC" regulated in 2006/72/EC directive states, for motorcycle with maximum speed lower than $140 \mathrm{~km} / \mathrm{h}$, the execution of two phases (WMTC_1 and WMTC_2). "Artemis Urban Cold" driving cycle was proposed within EU Artemis framework in order to study cold start influence on the exhaust emissions [14]: it includes 15 repetitions of a base module. During this experimental activity, measurements relative to "Artemis Urban Cold" were performed over three parts, each including 5 base modules. The main kinematic characteristics of all these driving cycles are summarized and reported in Table 3. 
Table 3. Kinematic characteristics of driving cycles adopted in the tests.

\begin{tabular}{lllll}
\hline & Duration [s] & $\begin{array}{l}\text { Length } \\
{[\mathrm{km}]}\end{array}$ & $\begin{array}{l}\text { Max. speed } \\
{[\mathrm{km} / \mathrm{h}]}\end{array}$ & $\begin{array}{l}\text { Average speed } \\
{[\mathrm{km} / \mathrm{h}]}\end{array}$ \\
\hline $\begin{array}{l}\text { ECE+EUDC } \\
\text { UDC_cold }\end{array}$ & 390 & 2 & 50 & 18.4 \\
UDC_hot & 780 & 4 & 50 & 18.4 \\
EUDC & 400 & 6.9 & 120 & 62.6 \\
WMTC & & & & \\
WMTC_1 & 600 & 4.1 & 60 & 24.3 \\
WMTC_2 & 600 & 9.1 & 95 & 54.6 \\
Artemis_Urban Cold & 945 & 5 & 44 & 19 \\
$\quad$ ECE-47 & & & & \\
ECE-47_cold & 448 & 3.1 & 45 & 25.1 \\
ECE-47_hot & 448 & 3.1 & 45 & 25.1 \\
\hline
\end{tabular}

Subsequently, in order to reduce the variables on which the emissive behaviour depends (the driving cycle characteristics, the additional mixture enrichment during the engine warm-up, the catalyst light-off and its associated conversion efficiency), experimental tests were carried out only in hot conditions, thus eliminating the influence of the cold start transient that, as it will be shown, has a sturdy consequence on $\mathrm{CO}$ and HC emissions. Successively, in order to better understand the emission results so obtained, the kinematic characteristics of the different driving patterns were investigated. First, all the driving cycles performed during the experimental test were fragmented into basic kinematic sequences, which are composed of speed-time values between two consecutive stops, under hot conditions of the engine and examined by using the online emission results. Subsequently, these elementary sequences, which describe different situations present in an urban environment, were characterized with two kinematic factors in order to better define the pertinent driving behaviours. These parameters are the average speed and the product of average speed and average acceleration of each elementary sequence. The values of this product, in effect, characterize the traction power per unit mass required to overcome the inertia of the vehicle, then this factor is rigorously linked to the emissions and fuel consumptions of the motorcycles.

\section{RESULTS AND DISCUSSION}

\section{Results on Type-Approval Driving Cycle in Cold and Hot Conditions}

Regulation approved for the Euro-3 phase of the Directive 97/24/CE introduced important improvement with reference to normalized cycle, including more stringent limits, an extra-urban phase and the evaluation of the cold start transient. In accordance with this regulation, three different phases were considered during the European Type-Approval driving cycle ("ECE+EUDC"). The first phase is the "UDC_cold" phase composed of two base urban modes, the second is the "UDC_hot" composed of the subsequent four base urban modes, and the third phase is an extra urban part ("EUDC").

In Fig. 2-4, average experimental emission values of the tested motorcycle, expressed as mass emitted per kilometre travelled, are reported (in red scale colour) for the three phases and for the whole cycle of five test repetitions, also to verify compliance with Euro-3 emission limits. In these figures, the experimental results are reported also as average values of each phase and of the whole cycle, with the relevant standard 
deviations (to the average values), in order to evaluate the variability of tests in the laboratory. This motorcycle complies with the Euro-3 limits for $\mathrm{CO}, \mathrm{NO}$ and $\mathrm{HC}$ : in fact, $\mathrm{CO}$ emission factors in the complete cycle ranged between 0.96 and $2.26 \mathrm{~g} / \mathrm{km}, \mathrm{HC}$ varied between 0.12 and $0.16 \mathrm{~g} / \mathrm{km}$, and $\mathrm{NO}_{\mathrm{X}}$ between 0.11 and $0.22 \mathrm{~g} / \mathrm{km}$.

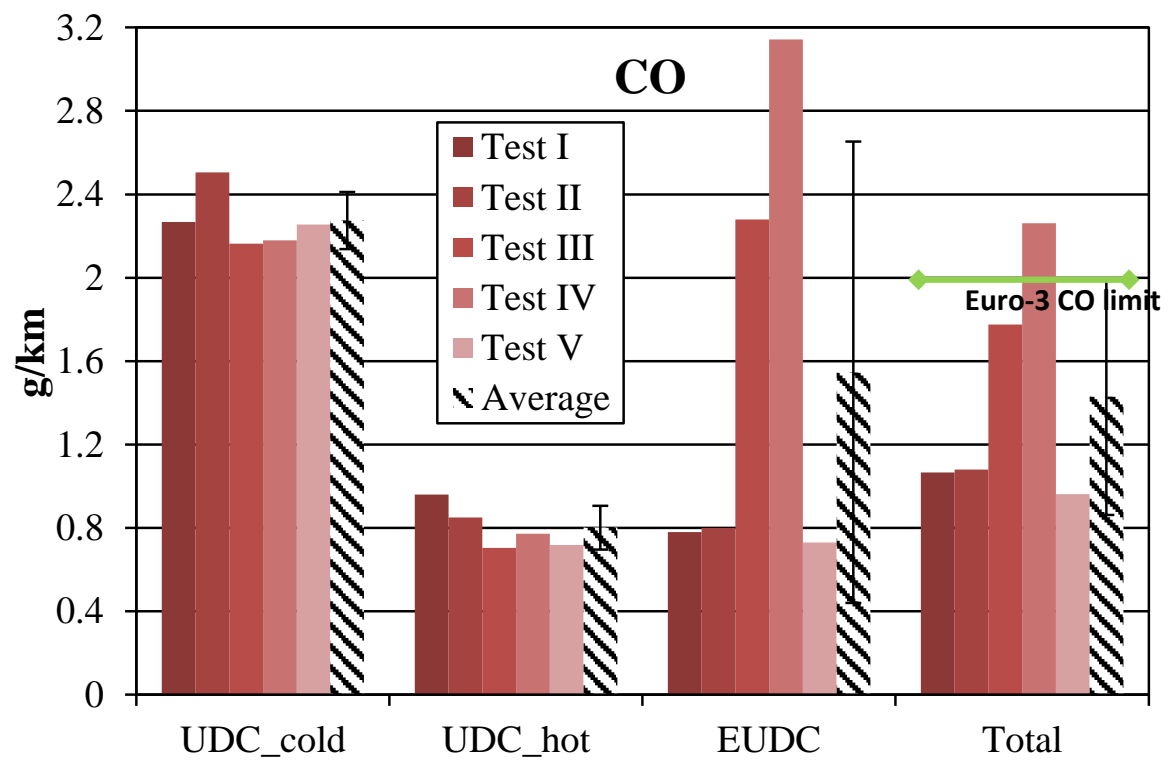

Figure 2. CO emission factors in "ECE+EUDC" phases and their variations to the average values.

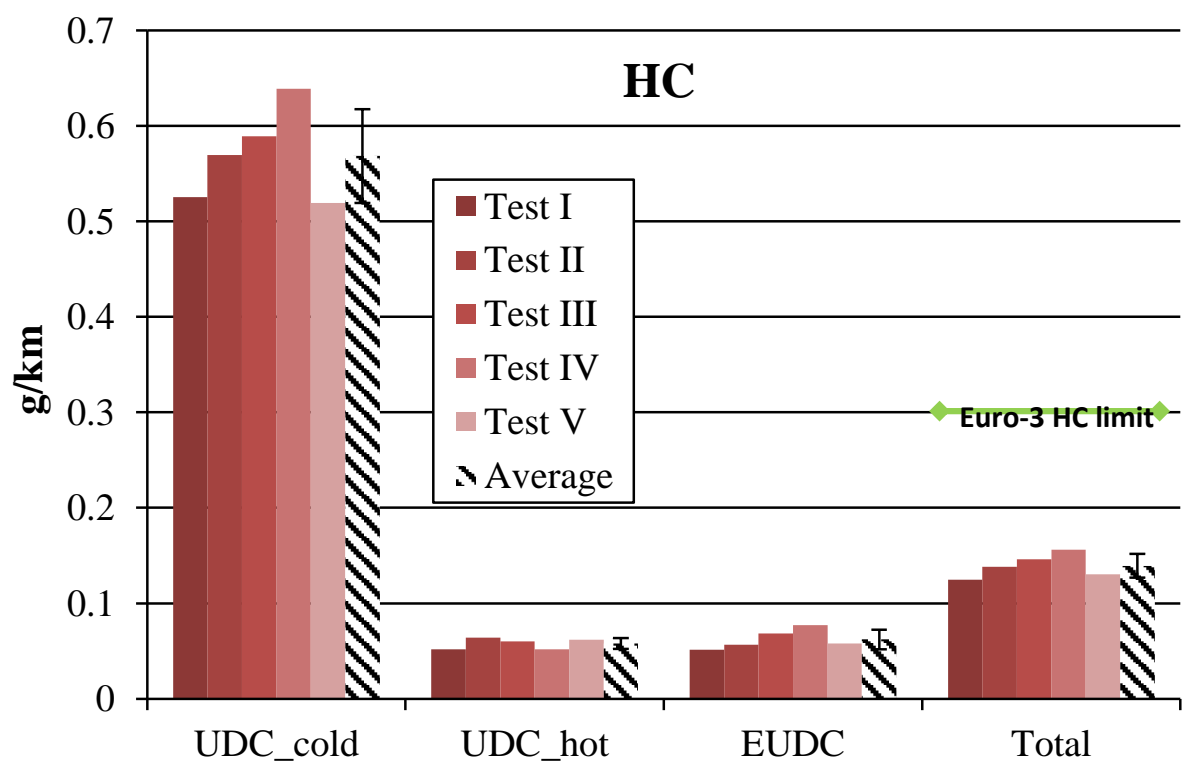

Figure 3. HC emission factors in "ECE+EUDC" phases and their variations to the average values.

As shown in Figure 2, CO emission factor obtained during the "EUDC" phase and the pertinent variation to the average $(\approx 72 \%)$ was much higher than values of "UDC hot" phase. This was due to the not completely accurate repeatability of some experimental tests performed by a human driver which failed to properly follow, for those tests, the 
whole speed-time profile of the driving cycle; besides, when running close to the maximum speed of the motorcycle (around $125 \mathrm{~km} / \mathrm{h}$ ), the engine operates at full load. In these circumstances, and for the particular electronic power management of the motorcycle, open loop operating conditions occurred during the "EUDC" phase of Test III and Test IV.

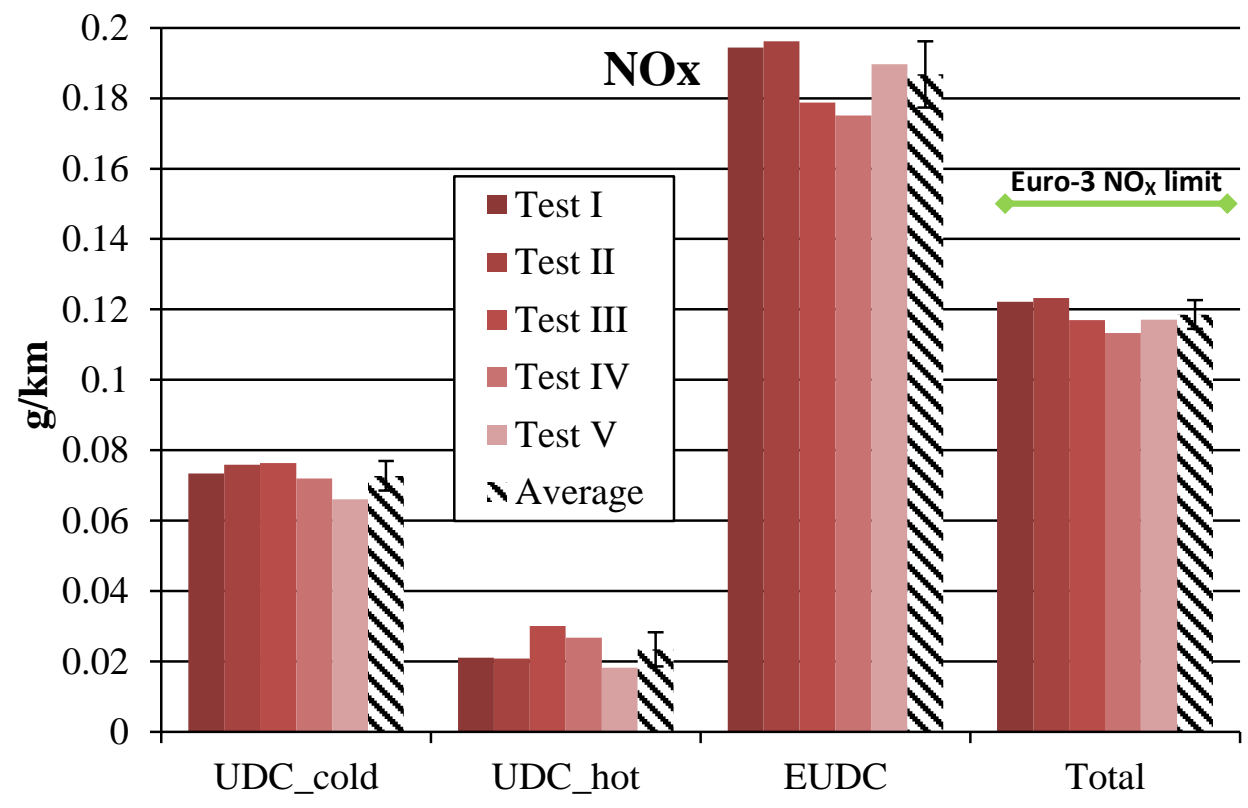

Figure 4. $\mathrm{NO}_{\mathrm{X}}$ emission factors in "ECE+EUDC" phases and their variations to the average values.

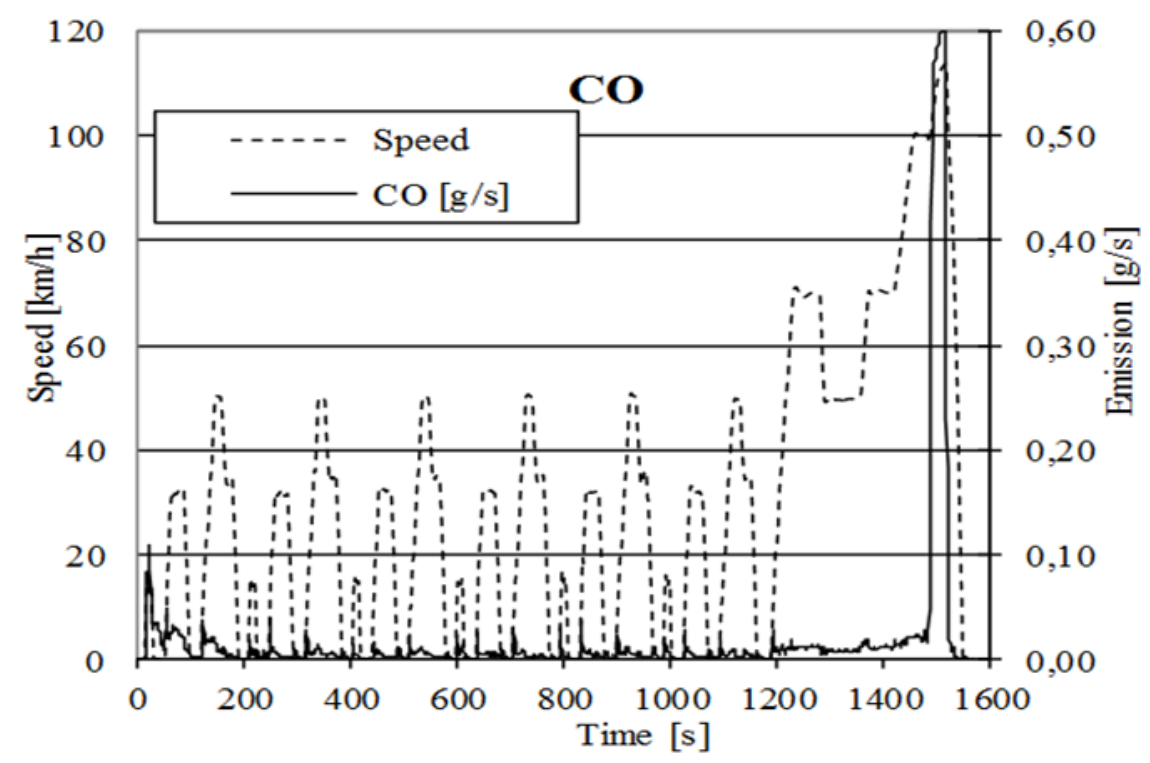

Figure 5. CO emissions and real speed during "ECE+EUDC" test IV.

To better explain this remark, the values of $\mathrm{CO}$ continuous experimental emissions and of real speed profile relevant to Test IV are shown in Figure 5; it is demonstrated that the instantaneous emission factor reaches a rapid and very high peak of around $0.6 \mathrm{~g} / \mathrm{s}$. The electronic mixture control of the vehicle, in fact, in order to offer definite driving 
dynamics characterized by high speed $(110 \mathrm{~km} / \mathrm{h})$ and acceleration, fixes a very rich airfuel mixture, external to the optimal range of catalyst efficiency, excluding the lambda sensor control and thus involving a considerable rise in $\mathrm{CO}$ emissions. On the other hand, the high percentage variance coefficient of HC emissions noticed in the UDC cold phase (Figure 3) was due to the difficulties found in the repeatability of tests in laboratory with different ambient conditions that influence the emission results during the cold transient time [15]. In Figure 5, it is also evident that the vehicle did not reach the maximum speed of the driving cycle, despite that the declared maximum speed of this motorcycle is even higher (Table 2). Another important aspect that can be observed among these experimental results is the differences between emissions during the cold phase and the hot phase of the Type-Approval driving cycle, as a consequence of the use of improved engines, in combination with the use of catalytic converter. During the hot phases (hot engine and completely efficient catalytic converter), emission levels of $\mathrm{CO}$ and $\mathrm{HC}$ were respectively about $60 \%$ and $90 \%$ lower than those detected during the cold start transient [16]. During the warm engine conditions, in fact, the catalyst and the engine are not at their best functioning conditions due to the rich gasoline content in the air-fuel mixture, and without attaining the light-off temperature of the the catalyst, this motorcycle produces high levels of $\mathrm{CO}$ and $\mathrm{HC}$ emissions [17].

For the last generation motorcycles that are equipped with a catalyst and electronic mixture control, emissions during the cold-start transient, therefore, are a significant fraction of total emissions, with a noticeable effect on air quality state [18]. As motorized two-wheelers are generally driven in urban contexts, it is evident that the cold-start effect must be taken into heedful consideration in evaluating emissions of $\mathrm{CO}$ and $\mathrm{HC}$ attributable to this vehicle category [19]. The influence of the cold start transient is undoubtedly less manifested on $\mathrm{NO}_{\mathrm{X}}$ emissions.

\section{Emissive Behaviour under Real Urban Conditions}

Average experimental emission factors, expressed as mass released of the regulated pollutants per kilometre, were detected and shown in the previous figures for cold and hot phase of the "UDC+EUDC" driving cycle. Nevertheless, these measured values are not effectively characteristic of real driving conditions, which are meaningfully dissimilar from the speed-time profile of the Type-Approval test cycle. This aspect is related to the kinematic parameters of the "ECE+EUDC" driving cycle that has the shortcoming of underestimating cycle dynamics, in fact, the relevant speed-time profile presents only one level of constant acceleration and a significant percentage of the driving cycle is characterized by constant speed. Besides, current emission models available in Europe are based mainly on the average trip speed; therefore, they underestimate the importance of vehicles instantaneous speed and acceleration.For all these reasons, it is necessary to obtain a more comprehensive valuation of the motorcycle emissive behaviour by assessing the emissions on other driving cycles; further experimental tests during realworld driving cycles are necessary in order to evaluate emissions under real-world motorcycle riding. In this study, two real urban driving cycles were considered: the "WMTC" and the "Artemis Urban Cold". Experimental emission factors of each driving cycle with their average speeds are shown in Table 4. It can clearly be observed that CO and $\mathrm{HC}$ emission factors calculated on the "Artemis Urban Cold" driving cycle are higher than those measured during the other driving cycles, because this cycle is characterized by many acceleration phases of higher levels than the others. Also, for similar values of average speed among the several test cycles, in fact, higher levels of acceleration of the "Artemis Urban Cold" correspond to an increase in energy request for the execution of 
the pertinent driving patterns, with consequent enrichment of the air-fuel mixture (outside the optimum range of catalyst efficiency), and then with increased $\mathrm{CO}$ and $\mathrm{HC}$ emissions. On the contrary, $\mathrm{NO}_{\mathrm{X}}$ emissions increase at high speed (during "ECE+EUDC" and "WMTC" driving cycles). This result can be ascribed both to the increased combustion temperatures at higher loads and to the high exhaust mass flow that limit the residence time of the exhaust in the catalytic converter [20].

Table 4. Emission factors for the driving cycles considered and data of ARTEMIS project WP 500.

\begin{tabular}{llll}
\hline $\begin{array}{l}\text { Test Cycle } \\
\text { (average speed }[\mathrm{km} / \mathrm{h}])\end{array}$ & $\begin{array}{l}\mathrm{CO} \\
{[\mathrm{g} / \mathrm{km}]}\end{array}$ & $\begin{array}{l}\mathrm{HC} \\
{[\mathrm{g} / \mathrm{km}]}\end{array}$ & $\begin{array}{l}\text { NOx } \\
{[\mathrm{g} / \mathrm{km}]}\end{array}$ \\
\hline ECE+EUDC $($ a.s.=29,7) & 1,42 & 0,139 & 0,119 \\
WMTC (a.s.=37,5) & 0,73 & 0,110 & 0,102 \\
Artemis Urban Cold (a.s.=19) & 1,67 & 0,146 & 0,086 \\
ECE 47 (a.s.=25,1) & 0,89 & 0,151 & 0,044 \\
ARTEMIS WP 500 & $4,04 \div 6,17$ & $0,26 \div 0,46$ & $0,094 \div 0,11$ \\
\hline
\end{tabular}

Table 4 also shows the emission factors calculated in the ARTEMIS WP 500 model by employing the emission functions (1) of the pertinent vehicular category, and by entering with speed values equal to the average speeds of the considered four driving cycles; the range of ARTEMIS estimations is relevant to the minimum and maximum value obtained with these four average speeds. Experimental emission factors of the same pollutants are then compared to these calculated values. It is noticed that $\mathrm{CO}$ and $\mathrm{HC}$ emission factors measured on the tested vehicle were always lower than ARTEMIS WP500 values, and these differences must be related to the vehicle fleet considered for the development of the emission database of the considered model. A decreasing enrichment for the tested vehicles might be the effect of internal engine optimization and more precise mixture control of fuel injection systems, consenting an improved control of fuel feeding and also increasing catalyst efficiency; on the other hand, the poor combustion quality occurring in four-strokes motorcycle engines, not fine-tuned in terms of air-fuel ratio, is probably responsible for high carbon monoxide and unburned hydrocarbons emission results of ARTEMIS WP500 measurement programme [21]. In this regard, since there are many parameters that influence emission levels (engine displacement, after-treatment systems, fuel injection systems, etc.), a really representative fleet should be composed of a large number of vehicles, thus leading to a great effort in executing experimental campaign, data processing and result analysis.

In order to explain the above emission results for each pollutant, a number of different aspects have to be considered, which are closely related: the driving cycle characteristics (in particular the acceleration phases with the associated rich values of airfuel ratio), the further mixture enrichment during the engine warm-up, the catalyst lightoff and its related conversion efficiency [22]. To lessen the number of all these variables on which the emissive behaviour depends, experimental tests, afterwards, were performed in hot conditions, thus removing the effect of the cold transient that, as analyzed above, has a strong effect above all on $\mathrm{CO}$ and $\mathrm{HC}$ emissions. In Figure 6, the hot emissions factors of $\mathrm{CO}, \mathrm{HC}$ and $\mathrm{NO}_{\mathrm{X}}$ are reported as a function of average vehicle speed for the different five hot phases of all the driving cycles, already described and considered in the previous paragraph. 

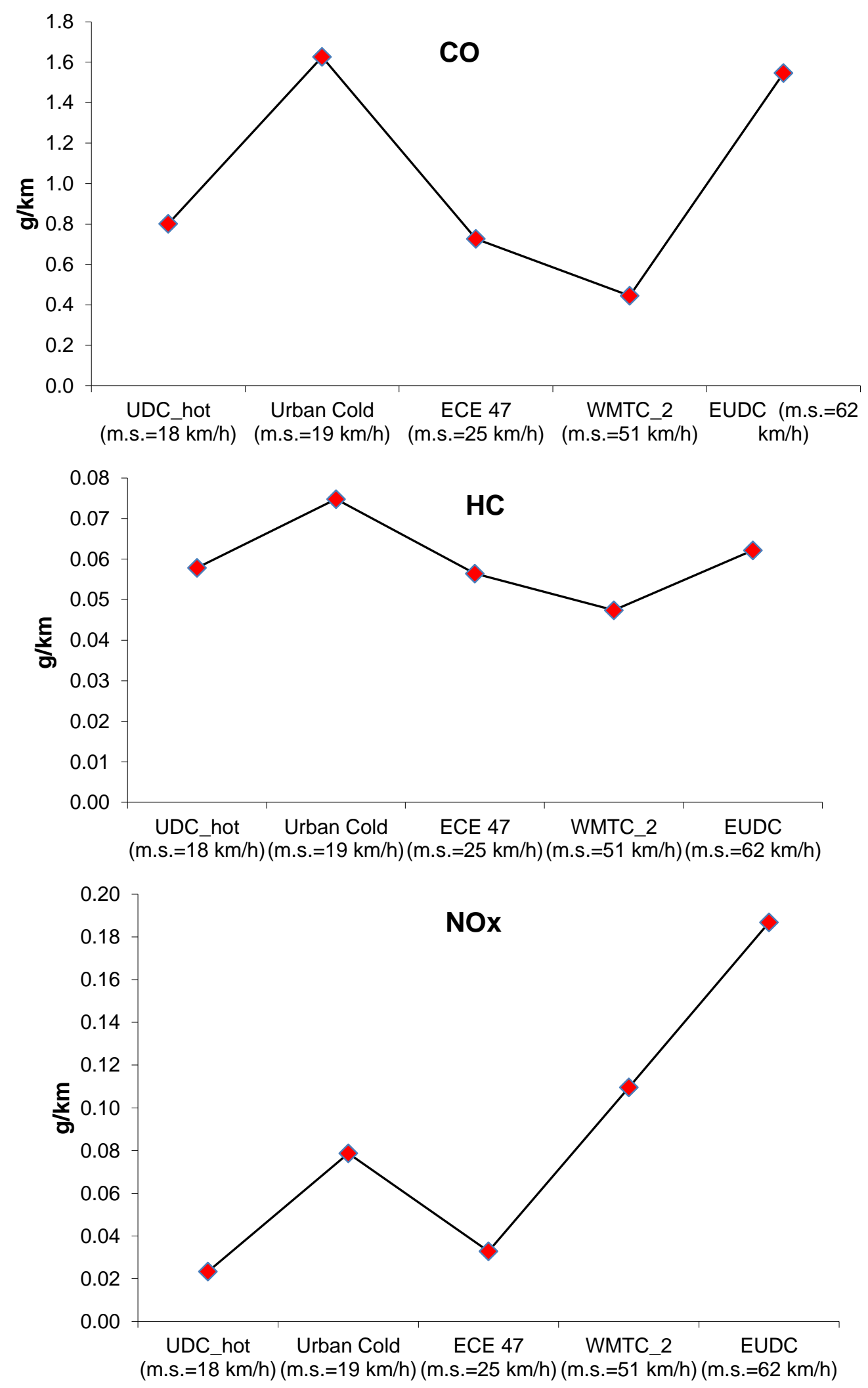

Figure 6. $\mathrm{CO}, \mathrm{HC}$ and $\mathrm{NO}_{\mathrm{X}}$ hot emission factors reported against average speed of each driving cycle. 

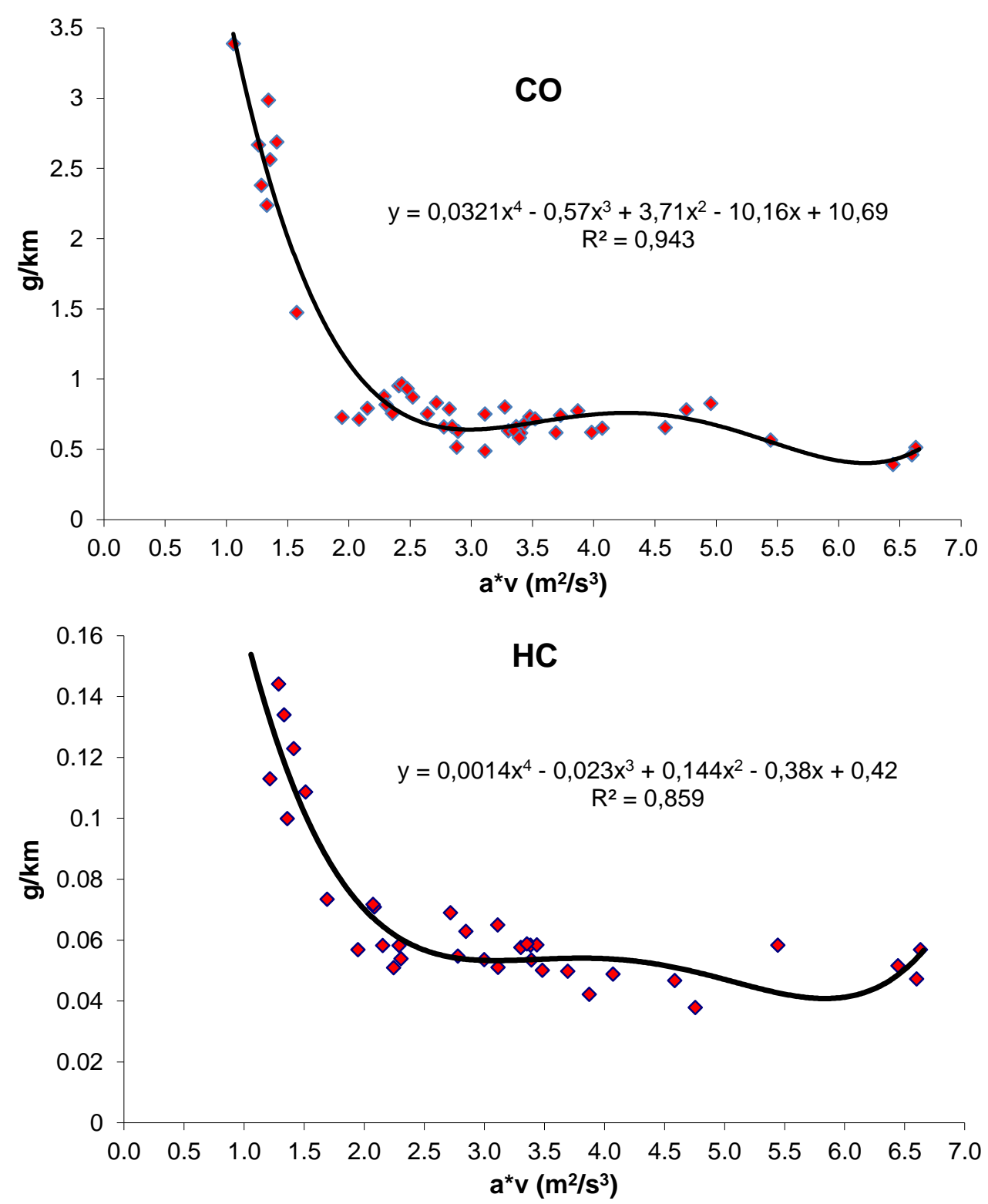

Figure 7. $\mathrm{CO}$ and $\mathrm{HC}$ hot emission factors measured against average product " $\mathrm{v} * \mathrm{a}$ " of each elementary test sequence.

Emission levels detected for real world cycle/speed profiles highlight a clear influence of the average speed, above all for $\mathrm{CO}$ and $\mathrm{NO}_{\mathrm{X}}$. However, for similar levels of average speed, emissions of $\mathrm{CO}, \mathrm{HC}$ and $\mathrm{NO}_{\mathrm{X}}$ calculated on the "Artemis Urban Cold" driving cycle (in the legend named as "Urban Cold") are higher than those measured during the "UDC_hot" phase. For CO and HC emissions, as described earlier, this could be ascribed to incomplete combustion in some driving situations during the "Artemis Urban Cold" characterized by a sudden steep increase in engine speed that is no longer compensated by the catalytic converter. In such driving situations, a rich fuel-air mixture could thus be assumed often to be provided to the combustion process of this motorcycle, which accounts for the very high emissions of $\mathrm{CO}$ and $\mathrm{HC}$ observed on the "Artemis Urban Cold" driving cycle. For this vehicle category, therefore, better performance with improved fuel economy and exhaust emissions are two different objectives, which are difficult to achieve together [23]. However, these differences are less pronounced for HC 
emissions because for lower engine loads, consequential to driving cycles ("UDC_hot" and "ECE 47") characterized by lesser and constant values of acceleration and by speedtime profiles with a considerable share at constant and low speed, the excessive leaning of the air-fuel mixture could cause irregular operating condition in the engine. This aspect compensates for the previous consideration and makes no great difference on $\mathrm{HC}$ emissions in hot conditions between the considered driving cycles [24]. About $\mathrm{NO}_{\mathrm{X}}$ emissions, the differences are due to excessive engine-temperatures produced by the frequent accelerations and decelerations that characterize particularly the "Artemis Urban Cold" driving cycle [25]. Then, to better compare these emission results, the kinematic characteristics of the different driving patterns must be examined. In order to better distinguish the several driving behaviours, in Figure $7 \mathrm{CO}$ and $\mathrm{HC}$ experimental emission factors acquired during numerous repetitions of the elementary test sequences, are linked to average product $v^{*} a$ of instantaneous speed and acceleration. As explained in the previous sessions, these basic kinematic sequences are speed-time profiles between two consecutive stops of the considered driving cycles (under warmed-up engine conditions and examined by employing the online emission results), while the average product $v^{*} a$ is a value connected to the emissions of the motorcycle because it characterizes the power per unit mass needed for the inertia of the vehicle. Examination of these values and of the pertinent trend lines, which cover different situations present on urban road, can recognize the driving sequences (then the driving patterns) that cause, for comparable levels of vehicle speeds, higher $\mathrm{CO}$ and $\mathrm{HC}$ emissions [26]. By using this statistical method, which considers the differences in the emissions for test cycles with comparable levels of average speeds but relatively high dissimilarities of other driving dynamics, the vehicles emission assessment is meaningfully improved if compared to the models and the methodologies that are mainly centred on average speed.

\section{CONCLUSIONS}

Two-wheelers are popular means of transportation in Europe, so their contribution to air pollution is usually significant, particularly in an urban environment. From this motivation, an experimental activity on motorcycles emissive behaviour was conducted by the Department of Industrial Engineering of the University of Naples. The present study aimed to determine the influence of several driving cycles on emissions of a medium-size motorcycle. In this study, the vehicle tested on a dynamometer bench is a motorcycle of $250 \mathrm{~cm}^{3}$ swept volume, equipped with a three-way catalyst and electronic mixture control. Statistical elaborations of the exhaust emissions of the regulated pollutants $\left(\mathrm{CO}, \mathrm{HC}\right.$ and $\left.\mathrm{NO}_{\mathrm{X}}\right)$ were performed both on the European Type-Approval cycle and on real-world cycles. Emission levels of this vehicle depend on considered driving cycle, due to the differences in kinematic parameters, such as speed and acceleration. All the experimental tests visibly indicate raised $\mathrm{CO}$ and $\mathrm{HC}$ cold additional emissions, if related with those obtained in hot conditions, since transient behaviour of the engine and catalytic converter, far from optimal working conditions, leads to meaningfully high levels of these pollutants. Regarding the hot emissions, the number and level of accelerations occurring during real world driving cycles compared to the Type-Approval driving cycle lead to a more marked variability of air-fuel ratio, which explains higher $\mathrm{CO}$ and $\mathrm{HC}$ measured emissions.

Some differences were observed between the experimental emission factors obtained on the tested motorcycle and the calculated values of ARTEMIS WP500 emission model. These differences are correlated to the characteristics of the vehicle fleet 
considered for the development of the emission database of the considered model.

Additional experimental studies will be performed, focusing on motorcycles belonging to different engine displacement and maximum speed classes, aiming at a broader assessment of the influence of numerous driving cycles on emissions of powered two wheelers under real conditions.

\section{ACKOWLEDGEMENTS}

The authors would like to thanks to University of Naples Federico II, Italy for their laboratories facilities and financial assistance.

\section{REFERENCES}

[1] Prati MV, Zamboni G, Costagliola MA, Meccariello G, Carraro C, Capobianco M. Influence of driving cycles on Euro 3 scooter emissions and fuel consumption. Energy Conversion and Management. 2011;52:3327-36.

[2] Iodice P, Senatore A. Exhaust emissions of new high-performance motorcycles in hot and cold conditions. International Journal of Environmental Science and Technology. 2015;12:3133-44.

[3] Iodice P, Senatore A. Appraisal of pollutant emissions and air quality state in a critical Italian region: methods and results. Environmental Progress \& Sustainable Energy. 2015;34:1497-505.

[4] Vasic A-M, Weilenmann M. Comparison of real-world emissions from two-wheelers and passenger cars. Environmental Science \& Technology. 2006;40:149-54.

[5] Prati MV, Costagliola MA. Emissions of fine particles and organic compounds from mopeds. Environmental Engineering Science. 2009;26:111-22.

[6] Ntziachristos L, Mamakos A, Samaras Z, Xanthopoulos A, Iakovou E. Emission control options for power two wheelers in Europe. Atmospheric Environment. 2006;40:4547-61.

[7] Hamada KI, Rahman M. An experimental study for performance and emissions of a small four-stroke SI engine for modern motorcycle. International Journal of Automotive and Mechanical Engineering. 2014;10:1852-65.

[8] Iodice P, Senatore A. Evaluation of dispersion models for predicting carbon monoxide concentrations from motor vehicles in a metropolitan area. International Review on Modelling and Simulations. 2013;6:1928-32.

[9] Iodice P, Senatore A. Experimental-analytical investigation to estimate an emission inventory from road transport sector. IAENG Transactions on Engineering Sciences: Special Issue of the International MultiConference of Engineers and Computer Scientists 2013 and World Congress on Engineering 2013: CRC Press; 2014. p. 141-9

[10] Gkatzoflias D, Kouridis C, Ntziachristos L, Samaras Z. COPERT 4: computer programme to calculate emissions from road transport - user manual (version 9.0). . European Environment Agency. 2012.

[11] Elst D, Gense N, Vermeulen R, Steven H. Artemis WP500 (powered two-wheelers emissions) - final report. EU project ARTEMIS, deliverable 5. TNO Automotive, Delft, The Netherlands. 2006.

[12] Steven H. Update der Emissionfaktoren für Motorräder. RWTÜV Fahrzeug GmbH Essen, im Auftrag von INFRAS Bern, Essen. 2003.

[13] Ajtay D, Weilenmann M, Soltic P. Towards accurate instantaneous emission models. Atmospheric Environment. 2005;39:2443-9. 
[14] André M. The ARTEMIS European driving cycles for measuring car pollutant emissions. Science of The Total Environment. 2004;334:73-84.

[15] Bozza F, Gimelli A, Fontanesi S, Severi E. 1D and 3D CFD Investigation of burning process and knock occurrence in a gasoline or CNG fuelled two-stroke SI engine. SAE Technical Paper; 2011.

[16] Roberts A, Brooks R, Shipway P. Internal combustion engine cold-start efficiency: A review of the problem, causes and potential solutions. Energy Conversion and Management. 2014;82:327-50.

[17] Iodice P, Prati MV, Senatore A. A calculation procedure for the evaluation of cold emissive behavior of high-performance motorcycles. SAE Technical Paper; 2011.

[18] Iodice P, Senatore A. Influence of ethanol-gasoline blended fuels on cold start emissions of a four-stroke motorcycle. Methodology and results. SAE Technical Paper; 2013.

[19] Iodice P, Senatore A. Cold start emissions of a motorcycle using ethanol-gasoline blended fuels. Energy Procedia. 2014;45:809-18.

[20] Alvarez R, Weilenmann M, Favez J-Y. Assessing the real-world performance of modern pollutant abatement systems on motorcycles. Atmospheric Environment. 2009;43:15039.

[21] Tsai J-H, Hsu Y-C, Weng H-C, Lin W-Y, Jeng F-T. Air pollutant emission factors from new and in-use motorcycles. Atmospheric Environment. 2000;34:4747-54.

[22] Zamboni G, Carraro C, Capobianco M. On-road instantaneous speed measurements on powered two-wheelers for exhaust emissions and fuel consumption evaluation. Energy. 2011;36:1039-47.

[23] Abdullah NR, Shahruddin NS, Mamat R, Ihsan Mamat A, Zulkifli A. Effects of air intake pressure on the engine performance, fuel economy and exhaust emissions of a small gasoline engine. Journal of Mechanical Engineering and Sciences. 2014;6:949-58.

[24] Bozza F, Gimelli A, Andreassi L, Rocco V, Scarcelli R. 1D-3D analysis of the scavenging and combustion process in a gasoline and natural-gas fuelled two-stroke engine. SAE Technical Paper; 2008.

[25] Kumar R, Durai BK, Saleh W, Boswell C. Comparison and evaluation of emissions for different driving cycles of motorcycles: A note. Transportation Research Part D: Transport and Environment. 2011;16:61-4.

[26] Iodice P, Prati MV, Senatore A. Emissive behaviour of two-wheeler vehicle category. Methodologies and results. Urban Environment: Alliance for Global Sustainability Bookseries 19; 2012. p. 181-90. 